\title{
Simulation of Absolute Amplitudes of Ultrasound Signals Using Equivalent Circuits
}

\author{
Jonny Johansson, Pär-Erik Martinsson, and Jerker Delsing
}

\begin{abstract}
Equivalent circuits for piezoelectric devices and ultrasonic transmission media can be used to cosimulate electronics and ultrasound parts in simulators originally intended for electronics. To achieve efficient systemlevel optimization, it is important to simulate correct, absolute amplitude of the ultrasound signal in the system, as this determines the requirements on the electronics regarding dynamic range, circuit noise, and power consumption.

This paper presents methods to achieve correct, absolute amplitude of an ultrasound signal in a simulation of a pulse-echo system using equivalent circuits. This is achieved by taking into consideration loss due to diffraction and the effect of the cable that connects the electronics and the piezoelectric transducer. The conductive loss in the transmission line that models the propagation media of the ultrasound pulse is used to model the loss due to diffraction. Results show that the simulated amplitude of the echo follows measured values well in both near and far fields, with an offset of about $10 \%$. The use of a coaxial cable introduces inductance and capacitance that affect the amplitude of a received echo. Amplitude variations of $60 \%$ were observed when the cable length was varied between $0.07 \mathrm{~m}$ and $2.3 \mathrm{~m}$, with simulations predicting similar variations.

The high precision in the achieved results show that electronic design and system optimization can rely on system simulations alone. This will simplify the development of integrated electronics aimed at ultrasound systems.
\end{abstract}

\section{INTRODUCTION}

Gimulation tools originally intended for electronic cirScuits (e.g., SPICE, PSpice) have become established in the design of ultrasound systems through the introduction of equivalent circuits. Specifically, they can be used to make system-level simulations, including both piezoelectric devices and electronics. Equivalent circuits for piezoelectric elements were initially developed in [1]-[3]. Leach further improved these models [4] by introducing controlled sources in order to avoid the use of negative capacitances and frequency-dependent transformers, which were used in early models. Mechanical losses occurring in the piezoelectric ceramic was introduced into the models by Püttmer et al. [5], who used it to model low-Q thickness mode transducers. Viscoelastic loss of the propagating wave also was modeled by the same methodology. The

Manuscript received September 30, 2005; accepted March 18, 2007. The authors want to acknowledge the knowledge and samples provided by Ferroperm A/S regarding piezoelectric ceramics. The work in this paper was funded by the European Union action in support of regional development within Objective One.

The authors are with the Department of Computer Science and Electrical Engineering, Luleå University of Technology, SE-971 87 Luleå, Sweden (e-mail: jonny.johansson@ltu.se).

Digital Object Identifier 10.1109/TUFFC.2007.491 effect of material properties on viscoelastic loss was further investigated by van Deventer et al. [6] to improve the accuracy of the models regarding loss and wave speed.

One targeted usage area for theses models is to be able to optimize an electronic-acoustic system with regard to, for example, dynamic range (DR), power consumption, and signal-to-noise ratio (SNR). In most cases, these parameters also are interconnected in that an increased design requirement in dynamic range or SNR will require an increase in power consumption. Both the dynamic range and the SNR will require the knowledge of two important properties of the system: first, the system noise level that in many cases is set by the electronics, and second, the amplitude of an incoming signal for various system configurations. The intended simulators generally perform well in simulations of electronic noise, providing that correct device models are used. Thus, if the absolute amplitude of a received acoustic signal in, for example, a pulse-echo system can be correctly simulated, the desired optimizations can be performed by simulations alone. This is particularly important when the electronics is designed into an integrated circuit, in which case each design cycle is very expensive.

This paper presents methods to achieve correct, absolute amplitude of an ultrasound signal in a simulation of a pulse-echo system using equivalent circuits. One important step to achieve this is to take into account the loss due to diffraction, which is a major contributor to acoustic loss in media with low viscoelastic attenuation. This is dealt with in the first part of the paper [7]. The second part of the paper considers the influence of parasitic components. The cable used to connect driver and receiver electronics with a transducer presents large parasitic inductance and capacitance in the system. This affects the amplitude of received echoes, as well as the shape of the excitation pulse [8]. Throughout the paper, simulations using equivalent circuit models together with chip-level electronics are compared to measurements. Because echo amplitudes are highly dependent on material parameters, a sensitivity analysis for the simulation model is presented.

\section{Diffraction Effects}

Diffraction loss (i.e., beam spreading) is a major loss contributor in low-loss media such as water. In this section we introduce a method to include diffraction loss in the equivalent circuits that model ultrasound devices. Thereafter, experiments and simulations are presented and compared. 


\section{A. Modeling Diffraction Loss}

Electrical transmission lines are used to model the propagation medium when an ultrasound system is simulated with equivalent circuits [5], [6]. Under the assumption of low-loss conditions $(R \ll \omega L, G \ll \omega C)$, the attenuation constant for an electrical transmission line can be written $[6]$ :

$$
\alpha=\frac{1}{2} \sqrt{L C}\left(\frac{R}{L}\right)+\frac{1}{2} \sqrt{L C}\left(\frac{G}{C}\right)
$$

here $R$ is resistance, $G$ is conductance, $C$ is capacitance, and $L$ is inductance per unit length of the transmission line. This can be rewritten:

$$
\alpha=\frac{1}{2} \frac{R}{Z_{0}}+\frac{1}{2} G Z_{0}
$$

where $Z_{0}=\sqrt{L / C}$ is the characteristic impedance of the transmission line.

A propagating sound wave is modeled as a forward travelling voltage wave in the transmission line. The amplitude of this voltage wave can be expressed as [9]:

$$
|V(z)|=\left|V_{0}\right| e^{-\alpha z},
$$

where $V_{0}$ is the voltage amplitude at $z=0$. Eq. (2) then gives:

$$
|V(z)|=\left|V_{0}\right| e^{-\left(R / 2 Z_{0}\right) z} e^{-\left(G Z_{0} / 2\right)} .
$$

Previous works regarding equivalent circuits have used $R$ to model viscous losses, and $G$ was set to 0 [5], [6]. An attenuation term that is nondependant of the attenuation caused by $R$ can be added by setting $G \neq 0$. In this paper, this is used to model the loss due to diffraction as:

$$
A_{\text {diff }}=\frac{|V(z)|}{\left|V_{0}\right| e^{-\left(R / 2 Z_{0}\right) z}}=e^{-\left(G Z_{0} / 2\right) z} .
$$

Solving for $G$ gives:

$$
G=\frac{2}{Z_{0} z} \ln \left(A_{\text {diff }}\right)
$$

which can be used as a parameter for the transmission line once the required diffraction loss $A_{\text {diff }}$ is known.

Given two identical transducers with radius $a$ spaced a distance $z$ apart, the diffraction loss from one to another is given by Kino [10] as:

$$
A_{\text {loss }}=\left|2 \int_{0}^{\infty} \frac{J_{1}^{2}(y)}{y} e^{j y^{2} S / r \pi} d y\right| .
$$

Here:

$$
S=z \lambda / a^{2},
$$

is the Seki parameter [11], and $\lambda$ is the wavelength of the transmitted ultrasound pulse. The integrand is $y=k_{r} a$ in which $k_{r}$ is the radial propagation constant. For a pulseecho system with a perfect plane reflector, $z$ in previous

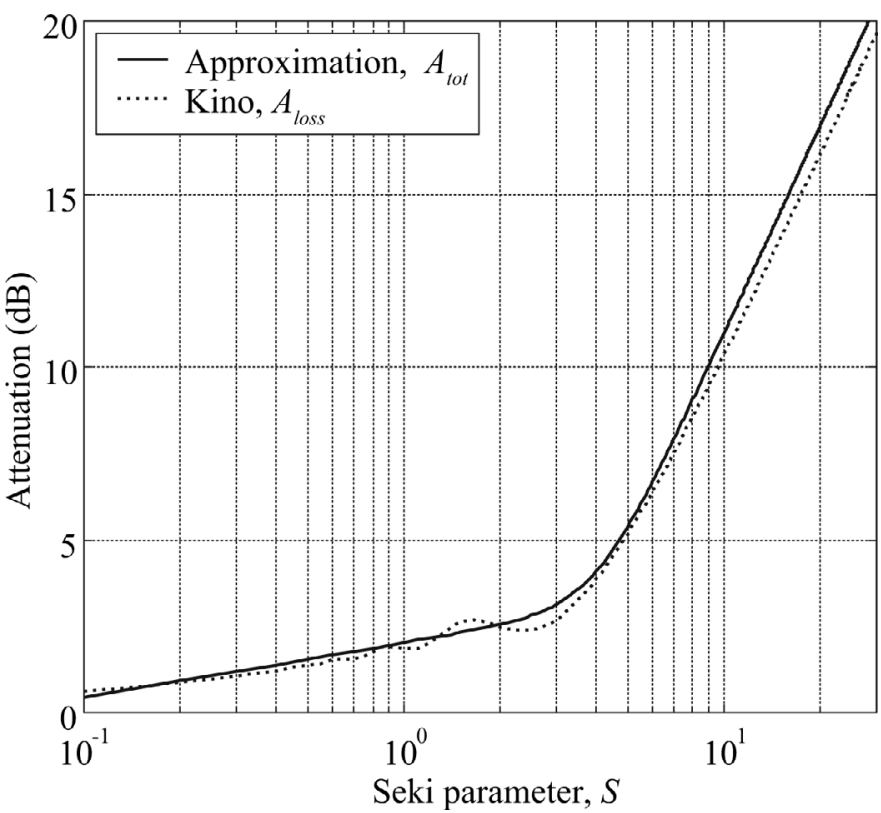

Fig. 1. Diffraction loss after Kino [10] versus approximation.

equations is replaced with $2 x$, where $x$ is the distance to the reflector. $A_{\text {loss }}$ as given by (7) is plotted for $0.1 \leq S \leq 30$ in Fig. 1. Eq. (7) must be solved numerically. Thus, it is difficult to use as a parameter in simulators like SPICE and PSpice. To arrive at an analytical approximative expression for use in the simulators, it can be noted in Fig. 1 that the behavior of (7) can be approximated with two straight lines in a log-log scale. Thus, an analogy with electrical filters was used to create an approximation of the diffraction loss. Two "filter" sections were used, one to create a straight line for $S \leq 4$ :

$$
A_{1}=\frac{1}{1.05(S / 0.1)^{0.08}}
$$

and one to increase the slope for $S \geq 4$ :

$$
A_{2}=\frac{1}{\left[(1+S / 4)^{5}\right]^{0.184}}
$$

Hence, the total attenuation can be expressed as:

$$
A_{\text {tot }}=A_{1} A_{2}
$$

The exponents for the denominators in (9) and (10) are chosen to give $A_{\text {tot }}$ a slope of $1 / S$ in the far field for $S>4$, which follows the Fraunhofer approximation. The order of the "filter section" in (10) is chosen to match the roll-off to the curve given by (7). The approximated diffraction loss value $A_{\text {tot }}$ is plotted for $0.1 \leq S \leq 30$ in Fig. 1. The small variations in attenuation for $S \leq 2$ are excluded in the approximation. If these were to be included, the model could be extended easily using more "filter sections" containing higher order terms of $S$. In this paper, they have been omitted for clarity. 


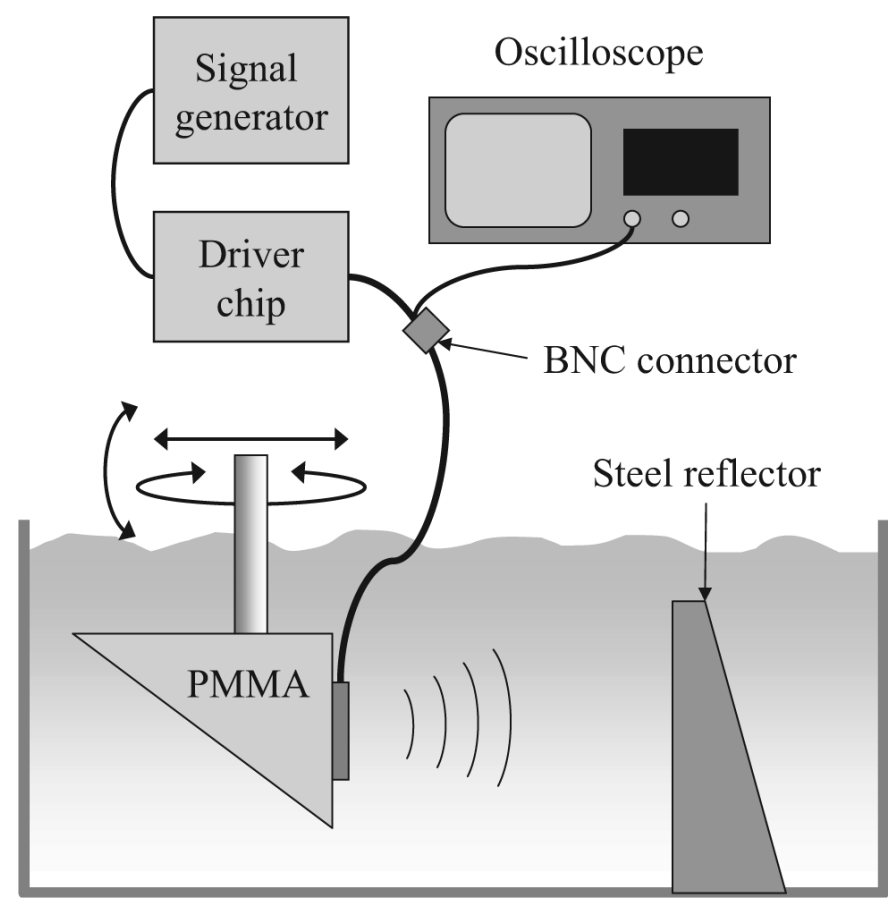

Fig. 2. Measurement setup for diffraction measurement.

Using (6) and (11) and setting $A_{\text {diff }}=A_{\text {tot }}$ now gives the conductance of the transmission line that models the propagation medium to be set as:

$$
\begin{aligned}
G=\frac{1}{Z_{0} x} \ln \left(\frac{1}{1.05\left(\left(2 x \lambda / a^{2}\right) / 0.1\right)^{0.08}}\right. \\
\left.\cdot \frac{1}{\left[\left(1+\left(2 x \lambda / a^{2}\right) / 4\right)^{5}\right]^{0.184}}\right) .
\end{aligned}
$$

Thus, we have arrived at a parameter that will model the diffraction loss in a pulse-echo system as a function of the distance to the reflector, $x$. The described method relies on the transducer radius $a$ as a starting value for beam radius and includes a near field in the calculations. Thus, it is applicable only if the wave propagation occurs mainly in one medium.

\section{B. Experimental Setup}

Diffraction measurements are performed in a pulse-echo setup in which the transducer is immersed in water as shown in Fig. 2. An ultrasound pulse is sent toward a steel reflector and received with the sending device. The piezoceramic element is a Pz27 disc manufactured by Ferroperm Piezoceramics A/S, Kvistgård, Denmark. The disc is mounted with cyanoacrylate glue on a carrier made of polymethyl methacrylate (PMMA) to form a transducer. The PMMA is used as backing, and it is shaped to reduce any echo returned to the rear side of the disc. The front side of the disc together with electrical connections is covered with one layer of $\mathrm{PC}-52$ protective lacquer (Henkel Loctite European Group, München, Germany) to allow immersion in water. The thin layer of lacquer does not significantly affect the measurements, thus it can be ignored
TABLE I

Simulation Data For Pz27.

\begin{tabular}{lll}
\hline Parameter & Notation & Value \\
\hline Radius & $a(\mathrm{~mm})$ & $3 / 6$ \\
Density & $\rho_{P Z}\left(\mathrm{~kg} / \mathrm{m}^{3}\right)$ & 7700 \\
Thickness & $l e n(\mathrm{~m})$ & $487 \cdot 10^{-6}$ \\
Permittivity & $\varepsilon^{S}(\mathrm{As} / \mathrm{V} \mathrm{m})$ & $8.09 \cdot 10^{-9}$ \\
Piezoelectric stress const. & $e^{33}\left(\mathrm{C} / \mathrm{m}^{2}\right)$ & 16 \\
Q-factor & $Q$ & 74 \\
Conductivity & $G(\mathrm{~S})$ & 0 \\
Elastic constant & $C^{E}(\mathrm{~Pa})$ & $1.13 \cdot 10^{11}$ \\
\hline
\end{tabular}

Data from Ferroperm A/S, Denmark, Feb. 2001,

in the simulations [12]. The transducer unit was mounted to a coordinate table that allows both translational and rotational motion. The transducer was adjusted to be parallel to the steel reflector by observing when the returned echo amplitude was maximized.

The transmit/receive electronics is a custom-made driver chip [13]. The chip is a push-pull driver that generates a square wave $5 \mathrm{~V}$ excitation pulse. The driver is controlled by a signal generator with the pulse width set to half the crystal oscillating period time, which yields maximum returned echo amplitude [14]. The driver chip is connected to the piezoelectric crystal with a $0.2 \mathrm{~m}$ long coaxial cable. Measurements of received echo signals are made on the driver side of the cable $\left(v_{D}\right)$ using a Tektronix TDS724 oscilloscope (Tektronix, Inc., Richardson, TX), which has an accuracy of $1 \%$ and a resolution of 8 bits.

\section{Simulation Setup}

The schematic used for simulation is shown in Fig. 3. Simulations were performed with the Cadence Spectre simulation engine (Cadence, San Jose, CA). Simulation of the electronics were made using transistor models from the semiconductor manufacturer, with estimated parasitic components included in the simulation schematic. The coaxial cable used to connect the electronics with the piezoelectric transducer was modeled as a lumped circuit. As discussed in Section III, the use of a correct cable model is vital to achieve correct absolute amplitudes in the system.

The temperature for the simulations was set to $20^{\circ} \mathrm{C}$. Parameter data for Pz27 used in the simulation are shown in Table I. Parameter data for PMMA, water, and steel are shown in Table II [6], [15].

\section{Diffraction Results}

1. Measurements: Measurements were performed for two different transducers with diameters of $6 \mathrm{~mm}$ and $12 \mathrm{~mm}$. The thickness of the discs is $0.5 \mathrm{~mm}$, which gives a resonance frequency of about $4 \mathrm{MHz}$. Results from the measurements are shown in Figs. 4 and 5. The far field limit in which the sound pulse starts a rapid divergence 


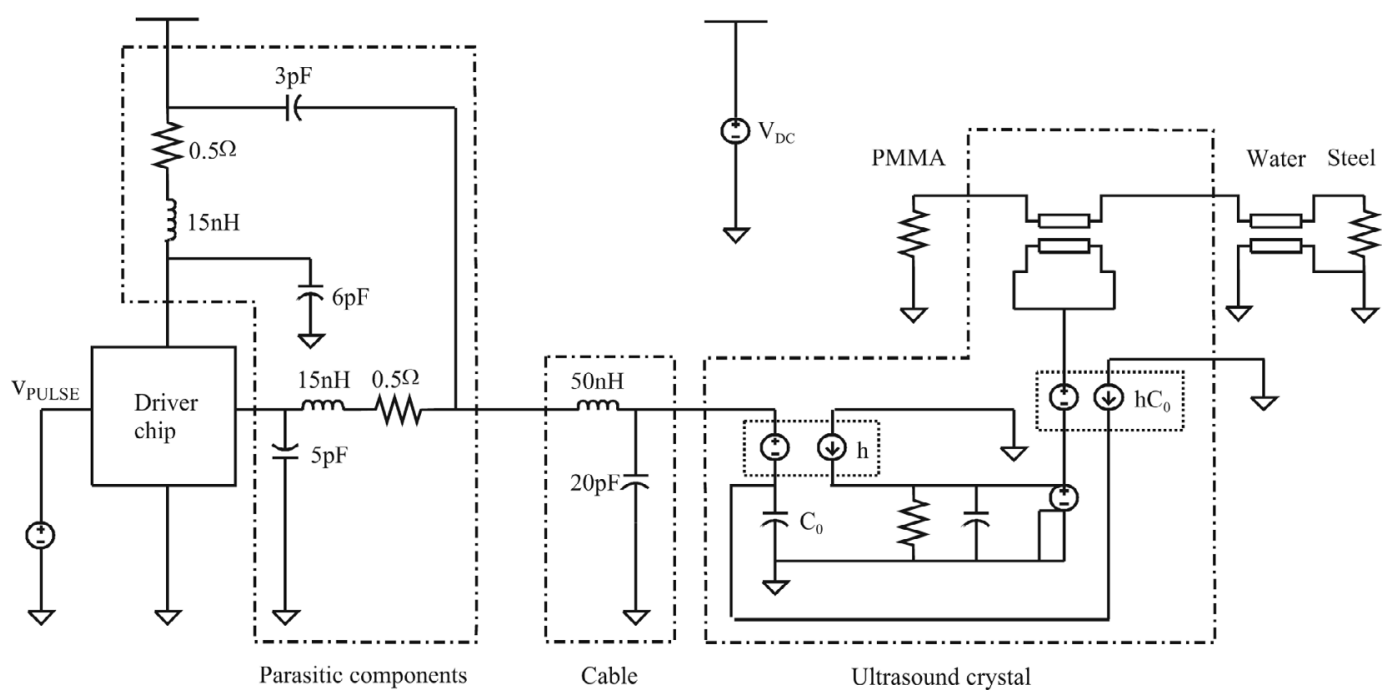

Fig. 3. Schematic used for simulation of diffraction effects.

TABLE II

Simulation Data for PMMA, Water, and Steel.

\begin{tabular}{lll}
\hline Parameter & Notation & Value \\
\hline Density of water & $\rho_{\text {wat }}\left(\mathrm{kg} / \mathrm{m}^{3}\right)$ & 998.2 \\
Speed of sound in water & $c_{\text {wat }}(\mathrm{m} / \mathrm{s})$ & 1478 \\
Viscous loss factor & $\alpha_{V}(\mathrm{~Np} / \mathrm{m})$ & 0.13 \\
Density of steel & $\rho_{\text {st }}\left(\mathrm{kg} / \mathrm{m}^{3}\right)$ & 8000 \\
Speed of sound in steel & $c_{\text {st }}(\mathrm{m} / \mathrm{s})$ & 5900 \\
Density of PMMA & $c_{\text {PMMA }}\left(\mathrm{kg} / \mathrm{m}^{3}\right)$ & 1190 \\
Speed of sound in PMMA & $c_{\text {PMMA }}(\mathrm{m} / \mathrm{s})$ & 2775 \\
\hline
\end{tabular}

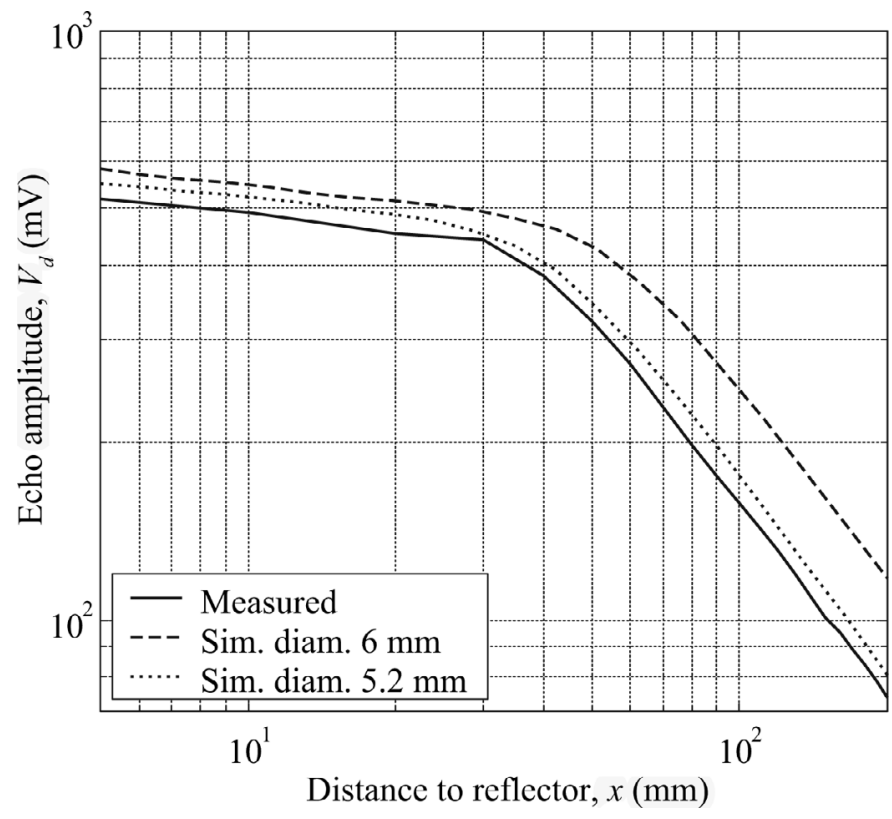

Fig. 4. Simulated versus measured echo amplitudes for transducer diameter $6 \mathrm{~mm}$.

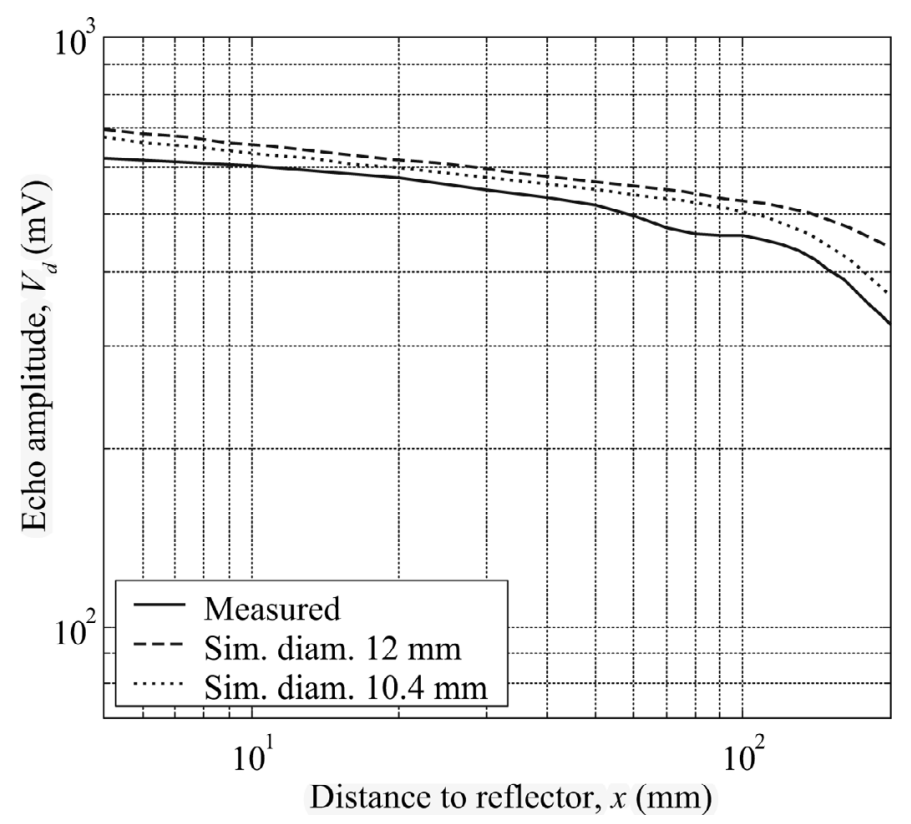

Fig. 5. Simulated versus measured echo amplitude for transducer diameter $12 \mathrm{~mm}$.

is clearly seen for the smaller transducer (Fig. 4) at a reflector distance of $30 \mathrm{~mm}$. The far field limit for the larger transducer (Fig. 5) is about $120 \mathrm{~mm}$. Water is a low-loss carrier of ultrasound waves, with attenuation due to viscoelastic loss of about $1.1 \mathrm{~dB} / \mathrm{m}$. This verifies that the measured attenuation profiles presented in Figs. 4 and 5 are indeed produced by diffraction effects.

2. Simulation: Initial simulations were made with the diameter of the piezoelectric disc set to $6 \mathrm{~mm}$ and $12 \mathrm{~mm}$. Results from these simulations are shown in Figs. 4 and 5 , respectively, together with the measured results. From the outcome of these simulations, it was clear that the diffraction loss was underestimated as the whole curve is shifted toward too high values along the $x$-axis. In the 
simulation model, the parameter $S$ as defined in (8) is decisive for the behavior of the curve. $S$ in turn is highly dependent on the transducer radius, $a$. If $a$ is decreased, the $S$ values will increase, and the curves will be shifted toward smaller values along the $x$-axis. Thus, the effective diameter in the model was decreased in order to find a better match to the measurements. From the initial results, it was seen that the curve should be shifted $25-30 \%$ to the left. This corresponds to a decrease in transducer diameter of $13-16 \%$. It was found that decreasing the diameter $13 \%$ gave a correct limit between near and far fields, leaving an amplitude offset of less than $10 \%$. Simulated results with disc diameters set to $5.2 \mathrm{~mm}$ and $10.4 \mathrm{~mm}$ are plotted in Figs. 4 and 5, respectively. The applied reduction in diameter agrees well with experimental data by Almqvist [16], who reports a measured effective diameter that is $14 \%$ smaller than the physical size of a transducer.

\section{PARAsitic Components}

Parasitic components are unwanted resistance, inductance, and capacitance occurring in cabling, bond wires, and circuit board paths. In order to accurately model the behavior of an electrical circuit, these components might need to be taken into account. To be able to include the parasitics in a simulation, the values of the components need to be estimated. On chip level, this can be done by the layout program used. The same is true for circuit boards. For bond wires that connect a chip to a socket approximate values can be achieved from the packaging facility.

Chip level capacitance and interconnect inductance are often in the $\mathrm{nH}$ and $\mathrm{pF}$ range as shown in Fig. 3. A coaxial cable that is used to connect the electronics with the piezoelectric transducer presents capacitance and inductance values that are considerably higher. Thus, this often will be a dominating source of parasitic inductance and capacitance in the system.

\section{A. The Coaxial Cable}

A simulation that uses the full model of a coaxial cable will achieve correct results with regard to the effect of the inductance and capacitance in the cable. A phenomenological explanation to the effects of the parasitic components arising in a coaxial cable, however, is easier to give if the lumped view is applied. Therefore, this section gives an introduction to the lumped model of a coaxial cable.

If the length of a coaxial cable is short compared to the wavelength of the propagating electromagnetic (EM) wave, the coaxial cable behaves as a lumped component consisting of a series inductor $L_{L}(\mathrm{H})$ and a parallel capacitance $C_{L}(\mathrm{~F})$. The limit for when to use a lumped model is often drawn when the length of the conductor exceeds $\lambda / 10$, where $\lambda$ is the wavelength of the EM wave. In this paper, the characteristic impedance of the coaxial cable is $50 \Omega$, which gives a capacitance of $100 \mathrm{pF} / \mathrm{m}$ and an inductance of $250 \mathrm{nH} / \mathrm{m}$ [17]. The propagation speed of an EM wave in a $50 \Omega$ cable is $c=2 \cdot 10^{8} \mathrm{~m} / \mathrm{s}$. With a wavelength of:

$$
\lambda=\frac{c}{f}
$$

the approximate upper frequency limit for when to use a lumped approach can be written:

$$
f_{\lim }=\frac{c}{10 l}
$$

The cable lengths used in this paper vary between $0.07 \mathrm{~m}$ and $2.32 \mathrm{~m}$, which gives a span for the frequency limit of:

$$
8.6 \mathrm{MHz} \leq f_{\lim } \leq 286 \mathrm{MHz}
$$

where the lower bound corresponds to the long cable. The center frequency of the transducer is $4.3 \mathrm{MHz}$, which leaves it below the limit throughout the range of cable lengths. Thus, for a first-order analysis of oscillation behavior such as discussed below, the lumped cable model can be used.

\section{B. Experiments and Simulations}

Simulations and measurements were performed to investigate the effects of varying cable lengths. The propagation medium used for these measurements was PMMA. The piezoelectric disc was mounted with cyanoacrylate glue to a 10-mm thick plate of PMMA, and air was used as backing. The echo was received from the PMMA/air interface opposite to the piezoelectric disc. Driver electronics as well as the type of piezoelectric element was the same as in the diffraction experiment, with a $12-\mathrm{mm}$ disc used in this case. The temporal behavior of the excitation pulse and the amplitude of the received echo were investigated. The excitation pulse was measured at the piezoelectric disc end of the cable $\left(v_{P}\right)$; amplitude of the received echoes was measured at the driver electronics $\left(v_{D}\right)$.

1. Excitation Pulse Shape: Cable lengths between $0.07 \mathrm{~m}$ and $2.06 \mathrm{~m}$ were used. Temporal behavior of the excitation pulse are shown in Figs. 6 and 7. It can be seen that simulations and measurements show very good agreement for both long and short coaxial cables. For short cable length (Fig. 6), an oscillation can be seen during the excitation pulse. This is due to the resonance circuit that is formed by the cable and bonding inductances in series with the static capacitance $C_{0}$ of the transducer. For the short cable length, the subsequent ringing of the transducer has very high content of harmonics and is almost triangular in shape. This is due to the high content of harmonics in the excitation pulse applied to the piezoelectric transducer.

For long cable lengths (Fig. 7), the inductance of the coaxial cable becomes significant. At a cable length $l=$ $2.07 \mathrm{~m}$ it is $L_{L}=518 \mathrm{nH}$. Furthermore, the capacitance of the coaxial cable which is $100 \mathrm{pF} / \mathrm{m}$ should be added to the capacitance in the oscillation circuit, yielding a total capacitance of $C_{T O T}=1.64 \mathrm{nF}$. Thus, the oscillation 


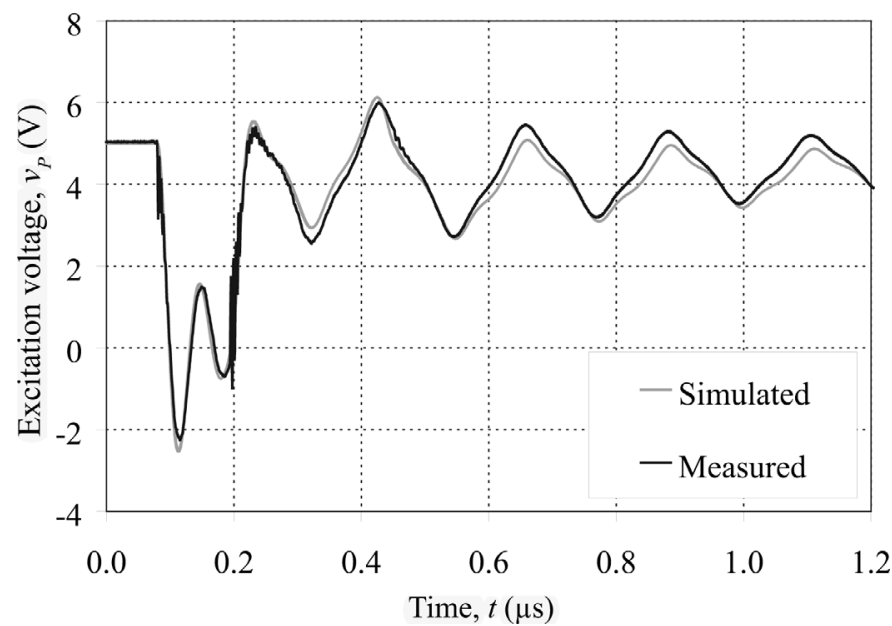

Fig. 6. Measured and simulated voltages at transducer for 7-cm coaxial cable.

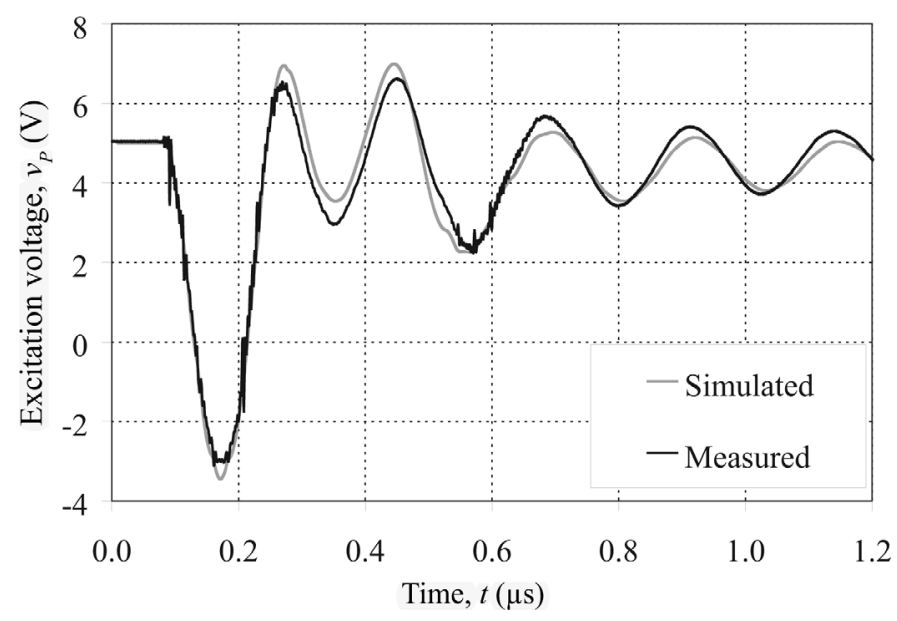

Fig. 7. Measured and simulated voltages at transducer for $2.06-\mathrm{m}$ coaxial cable.

frequency of the $L C$ circuit would be $5.5 \mathrm{MHz}$. The ringing during excitation is shown in Fig. 7. During the time of the excitation pulse, only one peak can be seen in the ringing that makes it hard to estimate the center frequency. However, it can be seen that it is of the order of magnitude as calculated above. The inductance and capacitance here form a low pass filter with a cut-off frequency around $5.5 \mathrm{MHz}$, which prevents fast transients to reach the transducer. This can be seen in the ringing of the transducer, which now shows a more sinusoidal shape due to reduced harmonic content, Fig. 7.

2. Received Echo: The peak-to-peak amplitude for the received echo was measured and simulated as a function of the cable length. Results are shown in Fig. 8. The simulations follow measured data fairly well, although a varying offset can be seen. One possible explanation to the variation of the offset is the presence of harmonics for short cable lengths as discussed in Section III-B,1. Due to the high frequency dependence of the attenuation in PMMA, these harmonics are filtered in the measurements [6]. The

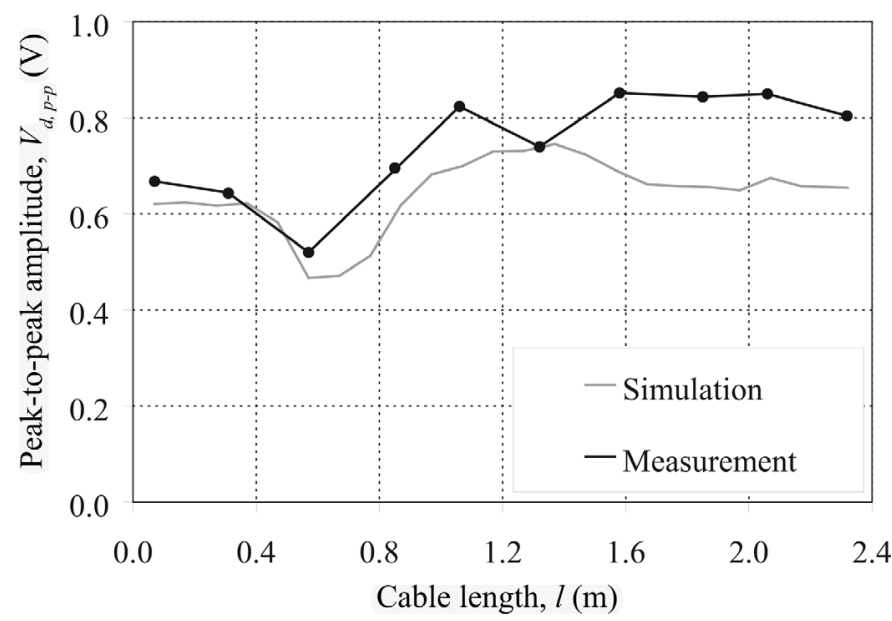

Fig. 8. Peak-to-peak voltage of received echo for varying length of coaxial cable.

harmonics in the simulation are not subject to this, as the frequency dependency is not modeled. Thus, the echoes received in the simulations have too high a content of overtones, which results in too high simulated peak-to-peak amplitudes for short cable lengths.

Amplitude variations up to $60 \%$ take place as the cable length is varied in both simulations and measurements. A qualitative explanation to the amplitude variation is the shift of the oscillation frequency of the $L C$ circuit as the cable length is varied. As this frequency varies, the oscillation will work in various phase positions versus the excitation pulse, thereby amplifying or attenuating the ringing of the transducer.

\section{Discussion}

As noted above, offsets remain between simulations and measurements. This may well be due to tolerances in material parameters, which normally are given to $\pm 10 \%$. Variations in material parameters also will affect the frequency and thereby the wavelength. This will change the Seki parameter $S$ and shift the diffraction curve along the $x$-axis as discussed above.

A simulated sensitivity analysis was performed to investigate the influence of variations in material parameters. Two distances were chosen, $5 \mathrm{~mm}$ (near field), and $200 \mathrm{~mm}$ (far field). The disc diameter used was $5.2 \mathrm{~mm}$. Parameter values were increased one by one with $10 \%$, and the resulting change in received echo amplitude was recorded. Although this simple analysis does not include correlation effects, it does give an indication of the relative influence of the parameters [18]. Results from the simulations are shown in Table III. It is seen that variations in material parameters can have a major effect on the received echo amplitude.

Uncertainties also exist in the simulation of the electronics. Variations in performance occur between produced batches of silicon chips. The manufacturer deals with this 
TABLE III

Result of Sensitivity Analysis.

\begin{tabular}{lcc}
\hline \multirow{2}{*}{ Parameter } & \multicolumn{2}{c}{ Change in echo amplitude $(\%)$} \\
\cline { 2 - 3 } & Near field & Far field \\
\hline$\varepsilon^{S}$ & -5.8 & -5.9 \\
$e^{33}$ & 14.4 & 15.6 \\
$Q$ & 1.2 & 1.5 \\
$\rho_{\text {PZ27 }}$ & -2.8 & -5.1 \\
$C^{E}$ & -8.7 & -5.5 \\
$Z_{\text {PMMA }}$ & -5.7 & -5.0 \\
$Z_{\text {Water }}$ & 6.2 & 6.5 \\
$Z_{\text {Steel }}$ & 0.2 & 0.7 \\
\hline
\end{tabular}

by giving the circuit designer models of the process in socalled "corners" [19]. These may give simulations with typical, fast, and slow performance of the used components. Furthermore, the temperature dependence of the circuit performance can be high. The simulations presented in this paper are run with performance set to typical-mean, and temperature set to $20^{\circ} \mathrm{C}$. To get a complete view of the system behavior under real operating conditions, simulations should be run in various corner settings and temperatures as recommended by the manufacturer of the chip. Another possibility is to use Monte Carlo simulations to randomly vary a number of parameters in the electronics.

\section{Conclusions}

By including effects due to diffraction and parasitic components, we have shown that it is possible to accurately simulate absolute amplitudes of ultrasound signals in pulse-echo systems using equivalent circuits.

The presented methods can be used either for systems using separate transmitter/receivers of equal size, or in a pulse-echo system. Excellent agreement has been shown between simulated and measured results for a pulse-echo system in water when diffraction effects are taken into account. Simulated echo amplitudes were within $10 \%$ of measured data in both near and far fields. Furthermore, the influence of the coaxial cable connecting the electronics and the piezoelectric transducer has been investigated. Pulseecho amplitude variations of $60 \%$ were observed when the cable length was varied between $0.07 \mathrm{~m}$ and $2.3 \mathrm{~m}$, with simulations predicting similar variations.

The high precision in the achieved results show that electronic design and system optimization can rely on sys- tem simulations alone. This has the potential to simplify the development of integrated electronics aimed at ultrasound systems.

\section{REFERENCES}

[1] W. P. Mason, Electromechanical Transducers and Wave Filters. New York: Van Nostrand, 1942.

[2] M. Redwood, "Transient performance of a piezoelectric transducer," J. Acoust. Soc. Amer., vol. 33, pp. 527-536, Apr. 1961.

[3] R. Krimholtz, D. A. Leedom, and G. L. Matthei, "New equivalent circuits for elementary piezoelectric transducers," Electron. Lett., vol. 6, pp. 398-399, 1970.

[4] W. M. Leach, "Controlled-source analogous circuits and SPICE models for piezoelectric transducers," IEEE Trans. Ultrason., Ferroelect., Freq. Contr., vol. 41, pp. 60-66, Jan. 1994.

[5] A. Püttmer, P. Hauptmann, R. Lucklum, O. Krause, and B. Henning, "SPICE model for lossy piezoceramic transducers," IEEE Trans. Ultrason., Ferroelect., Freq. Contr., vol. 44, pp. 60-66, Jan. 1997.

[6] J. van Deventer, T. Löfqvist, and J. Delsing, "PSpice simulation of ultrasonic systems," IEEE Trans. Ultrason., Ferroelect., Freq. Contr., vol. 47, pp. 1014-1024, July 2000.

[7] J. Johansson and P.-E. Martinsson, "Incorporation of diffraction effects in simulations of ultrasonic systems using PSpice models," in Proc. IEEE Ultrason. Symp., 2001, pp. 405-410.

[8] J. Johansson and J. Delsing, "Effects of parasitic electrical components on an ultrasound system: Measurements and simulations using SPICE models," Proc. SPIE, vol. 4946, 2002, pp. $174-182$.

[9] R. Ludwig and P. Bretchko, RF Circuit Design. Upper Saddle River, NJ: Prentice-Hall, 2000.

[10] G. S. Kino, Acoustic Waves: Devices, Imaging and Analog Signal Processing. Englewoods Cliffs, NJ: Prentice-Hall, 1988.

[11] H. Seki, A. Granato, and R. Truell, "Diffraction effects in the ultrasonic field of a piston source and their importance in the accurate measurement of attenuation," J. Acoust. Soc. Amer., vol. 28, pp. 230-238, Mar. 1956.

[12] M. Willatzen, "Ultrasound transducer modeling - Received voltage signals and the use of half-wavelength window layers with acoustic coupling layers," IEEE Trans. Ultrason., Ferroelect., Freq. Contr., vol. 46, pp. 1164-1174, Sep. 1999.

[13] J. Johansson, "Optimization of a piezoelectric crystal driver stage using system simulations," in Proc. IEEE Ultrason. Symp., 2000, pp. 1049-1054.

[14] J. Johansson and J. Delsing, "Microelectronics mounted on a piezoelectric transducer: Method, simulations, and measurements," Ultrasonics, vol. 44, pp. 1-11, Jan. 2006.

[15] C. Nordling and J. Österman, Physics Handbook. Lund, Sweden: Studentlitteratur AB, 1988.

[16] M. Almqvist, "High resolution light diffraction tomography: Nearfield measurements of $10 \mathrm{MHz}$ continuous wave ultrasound," Ultrasonics, vol. 37, pp. 343-353, June 1999.

[17] D. K. Cheng, Field and Wave Electromagnetics. Reading, MA: Addison-Wesley, 1989.

[18] G. E. P. Box, W. G. Hunter, and J. Hunter, Statistics for Experimenters: An Introduction to Design, Data Analysis, and Model Building. New York: Wiley, 1978.

[19] D. Foty, MOSFET Modeling with SPICE. Upper Saddle River, NJ: Prentice-Hall, 1997. 